\title{
Three-dimensional light bullets in heterogeneous medium of carbon nanotubes with metallic conductivity
}

\author{
M. B. Belonenko ${ }^{1,2}$, I. S. Dvuzhilov ${ }^{2}$, Yu. V. Nevzorova ${ }^{2}$, E. N. Galkina ${ }^{2,3}$ \\ ${ }^{1}$ Laboratory of Nanotechnology, Volgograd Institute of Business, \\ Yuzhno-Ukrainskaya ul. 2, Volgograd, 400048, Russia \\ ${ }^{2}$ Volgograd State University, Universitetskii pr. 100, Volgograd, 400062 Russia \\ ${ }^{3}$ Volgograd State Medical University, Pavshikh Bortsov Sq., Volgograd, 400131, Russia \\ mbelonenko@yandex.ru, dvuzhilov.ilya@volsu.ru,nevzorkina@yandex.ru, galkina@gmail.com
}

PACS 72.20.Ht, 42.65.Re

DOI $10.17586 / 2220-8054-2017-8-4-435-440$

\begin{abstract}
Theoretical investigation of the dynamics of three-dimensional few-cycle optical pulses (light bullets) in an inhomogeneous medium of carbon nanotubes with metallic conduction was performed. The stable propagation of pulses under research in accordance with inhomogeneous medium parameters was determined.
\end{abstract}

Keywords: carbon nanotube, few-circle optical pulses, heterogeneous medium.

Received: 27 October 2016

Revised: 20 June 2017

\section{Introduction}

The dynamics of three-dimensional few-cycle optical pulses propagating in an inhomogeneous medium with metallic carbon nanotubes (CNTs) have been theoretically investigated in this paper. It is well known that there are different types of CNTs (chiral, arm-chair, and zig-zag); additionally, the chiral nanotubes can be either semiconducting or metallic, corresponding to the numbers determined by the different methods of graphene rolling up into CNT. Since there is no energy gap in the electronic spectrum in metallic CNTs, effects similar to those observed in graphene layer are possible. The study of three-dimensional few-cycle optical pulse (light bullet) dynamics in different media is of great interest not only from a theoretical point of view, but is also important for practical applications [1-3]. Note that light bullets are not stable and spreading in vacuum or linear media due to dispersion impact. For stable propagation of such pulses, the nonlinear medium in which the effects associated with dispersion could be offset by the nonlinear effects is required [4-7]. From this point of view, CNTs are promising media for the three-dimensional few-cycle optical pulse propagation, since in CNTs, the nonlinearity is determined by nonparabolicity of the electron dispersion law which interact with the light pulse field [8-10]. In the above mentioned research the results were obtained for CNTs of the zig-zag type. Since we consider the interaction between the pulse's electric field and the CNT conduction band electrons, the analysis of the light bullet's stable propagation in the array of metallic CNTs due to the change in the electron dispersion law is absolutely non-trivial [11]. An increased focus on similar pulses is due to a wide range of practical applications, such as ultra-high resolution microscopy, data transfer via fiber-optic communication lines, micro- and nanoparticle manipulations, etc. [12-15]. The importance of practical applications and considerations mentioned above give considerable impetus to the present research.

\section{Basic equations}

The electromagnetic pulse propagation in carbon nanotube array has been considered, wherein both the pulse electric field and the current are directed along the nanotube axis. The geometry of the problem is presented in Fig. 1.

The Hamiltonian for electron system can be written in the form:

$$
H=\gamma \sum_{j \Delta \sigma} a_{j \sigma}^{+} a_{j+\Delta \sigma}^{+} h . c .
$$

where $a_{j \sigma}^{+}, a_{j \sigma}$ are creation and annihilation operators of electrons at site $j$ with spin $\sigma, \gamma$ is a hopping integral, determined by the electron wave function overlapping at adjacent sites. 


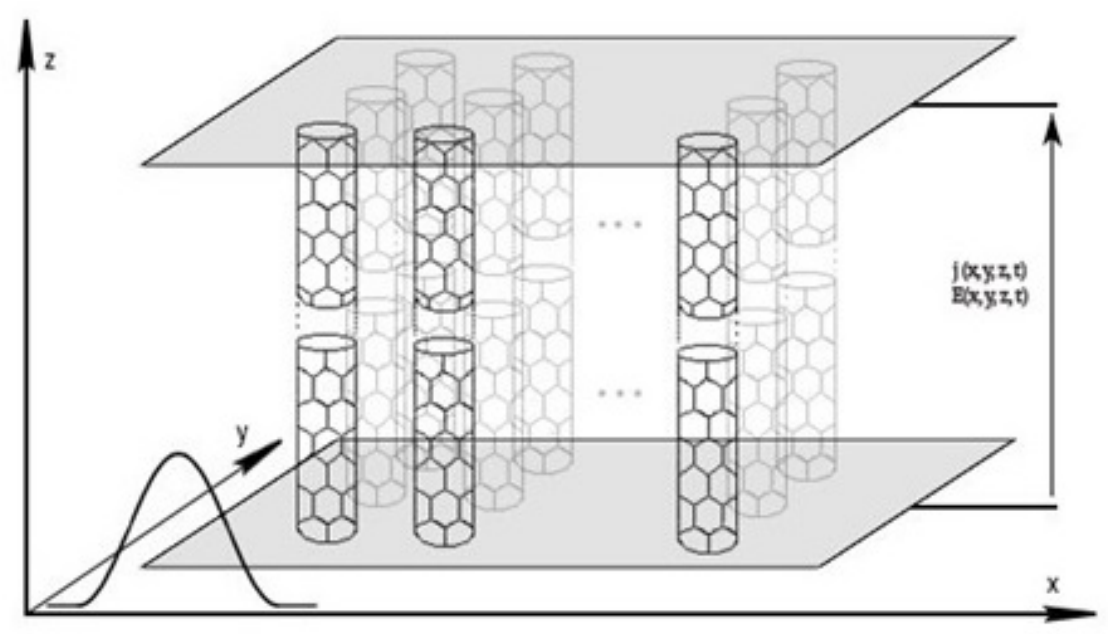

FIG. 1. Geometry of the problem, where $j(x, y, t)$ is the current along the CNT axis, $E(x, y, t)$ is the pulse electric field

Using the following Fourier series:

$$
\begin{aligned}
& a_{n \sigma}^{+}=\frac{1}{N^{1 / 2}} \sum_{j} a_{j \sigma}^{+} \exp (i j n), \\
& a_{n \sigma}=\frac{1}{N^{1 / 2}} \sum_{j} a_{j \sigma} \exp (-i j n),
\end{aligned}
$$

which diagonalizes the Hamiltonian $H$, thus making it easy to obtain the electron's spectrum describing the properties of electron subsystem in absence of the Coulomb repulsion $\varepsilon_{s}(p)$.

As a rule, the study of carbon nanotubes' electronic structure is carried out in framework of aanalysis of dynamics of $\pi$-electrons in strong-coupling approximation. The CNT radius is considered to be much smaller than characteristic dimension of light bullet that allows us to neglect the field spatial inhomogeneity in nanotubes:

$$
\varepsilon\left(k_{x, y}\right)= \pm \gamma_{0}\left(1+4 \cos \left(\frac{q \pi}{N_{x}}-\frac{N_{y} k a}{2 N_{x}}\right)+4 \cos ^{2}\left(\frac{q \pi}{N_{x}}-\frac{N_{y} k a}{2 N_{x}}\right)\right)^{1 / 2}
$$

where $\gamma_{0} \approx 2,7 \mathrm{eV}, k a$ belongs to the interval $[-\pi ; \pi], k$ is the wave vector along the CNT axis, $a=3 b / 2 \hbar$, $b=0.152 \mathrm{~nm}$ are the distances between adjacent carbon atoms. $N_{y} a$ corresponds to the translation operation, and $\sqrt{3} N_{x} a$ corresponds to the rotation operation.

Suppose that $N_{x}=N_{y}$. Thus, we get the dispersion law for CNT of arm-chair type with metallic conductivity. Note that $N_{x}$ is the number of hexagons along the circumference of carbon nanotubes.

A current arising in a metallic carbon nanotube is due to electrons which are described by the dispersion law for a branch crossing the Fermi level. The explored current is ballistic, i.e., flowing at times much shorter than the electron's relaxation times.

Using the standard Coulomb gauge [16] $\mathbf{E}=-\partial \mathbf{A} c \partial t$, the pulse electromagnetic field can be described by the Maxwell equations as follows:

$$
\frac{\partial^{2} \mathbf{E}}{\partial x^{2}}+\frac{\partial^{2} \mathbf{E}}{\partial^{2}}+\frac{\partial^{2} \mathbf{E}}{\partial z^{2}}-\frac{n^{2}(x, y, z)}{c^{2}} \frac{\partial^{2} \mathbf{E}}{\partial t^{2}}+\frac{4 \pi}{c} \mathbf{j}=0
$$

where $\mathbf{E}$ is the light pulse electric field, $j$ is the current density resulted from the pulse electric field action upon the CNT conduction band electrons, $t$ is time, $c$ is speed of light in the medium, $n(x, y, z)$ is the coefficient determining spatial variation of the carbon nanotube density.

The expression for the current density is given by:

$$
j=e \sum_{p s} v_{s}\left(p-\frac{e}{c} A(t)\right)\left\langle a_{p s}^{+} a_{p s}\right\rangle,
$$

where $v_{z}=\partial \varepsilon_{s}(p) / \partial p$, and the angle brackets denote an averaging with nonequilibrium density matrix $\rho(t)$ : $\langle B\rangle=\operatorname{Sp}(B(0) \rho(t))$. Due to the motion equation, we obtain: $\left\langle a_{p s}^{+} a_{p s}\right\rangle=\left\langle a_{p s}^{+} a_{p s}\right\rangle_{0}$, where $\langle B\rangle_{0}=\operatorname{Sp}(B(0) \rho(0))$. 
Expanding $v_{s}(p)$ in a Fourier series and summing up over $s$ and $p$, we get:

$$
\begin{aligned}
& j=-e n_{0} \sum_{k} B_{k} \sin \left(\frac{k e}{c} A(t)\right) \\
& B_{k}=\sum_{s=1}^{m} \int_{-\pi / a}^{\pi / a} d p A_{k s} \cos (k p) \frac{\exp \left(-\beta \varepsilon_{s}(p)\right)}{1+\exp \left(-\beta \varepsilon_{s}(p)\right)}
\end{aligned}
$$

where $n_{0}$ is the concentration of equilibrium electrons in carbon nanotubes, $\beta=1 / k T$,

$$
A_{k s}=\int_{-\pi / a}^{\pi / a} v_{s}(p) \sin (k p) d p
$$

are expansion coefficients decreasing with increasing $k$.

Finally, the nondimensionalized equation can be written in the form:

$$
\frac{\partial^{2} B}{\partial z^{2}}+\frac{1}{r} \frac{\partial}{\partial r}\left(\frac{\partial B_{z}}{\partial r}\right)-n(z, r) \sin (B)+\sum_{k=2}^{\infty} B_{k} n(z, r) \sin (k B)=0, r=\sqrt{x^{2}+y^{2}}
$$

Since there is a cylindrical symmetry, the summand, depending on the angle of rotation, tends to zero.

Note that equation (5) is a generalization of the well-known sine-Gordon equation.

Solving the problem, we neglect the diffraction spreading of the laser beam in direction along the CNT axis. We ignore the substrate electric field as well. Note that since both the typical size of CNTs and the distance between them are much smaller than the spatial domain typical size in which a few-cycle pulse is localized, we can use the continuous medium approximation and consider the current distributed over the volume.

\section{Results of numerical calculations}

Equation (5) under study has been solved numerically using the explicit finite difference cross-type schemes [17]. The time and coordinate steps were determined from standard stability conditions, and decreased until the solution changed in the eighth significant sign. Initial conditions for the vector potential of the light bullet electric field were given by the equations in Gaussian form:

$$
\begin{aligned}
& A(z, r, 0)=Q \exp \left(-\frac{\left(z-z_{0}\right)^{2}}{\gamma_{z}}\right) \exp \left(-\frac{r^{2}}{\gamma_{z}}\right) \\
& \frac{d A(z, r, 0)}{d t^{\prime}}=\left.\frac{2 Q v z}{\gamma^{2}}\left(-\frac{z-z_{0}}{\gamma_{z}}\right)^{2}\right|_{t^{\prime}=0} \exp \left(-\frac{r^{2}}{\gamma_{z}}\right),
\end{aligned}
$$

here $Q$ is pulse amplitude, $\gamma_{z}, \gamma_{r}$ are the parameters determining the pulse widths in $z$ - and $r$-direction respectively, and $v$ is the initial pulse velocity.

According to the numerical calculation results, the light bullet propagation is stable and the obtained evolution is presented in Fig. 2.

As can be seen from the figures, the solution for three-dimensional light bullet in an inhomogeneous nonlinear medium with metallic carbon nanotubes remains localized, but changes its spatial structure due to dispersion effects. The simultaneous impact of both the medium's nonlinearity and dispersion effects leads to complex structure formation on the pulse's trailing edge, which remains localized in a limited spatial domain.

The results of numerical simulation, depending on period of inhomogeneity are shown in Fig. 3. As expected, a few-cycle pulse propagates faster as the lattice constant increases. It is obvious, that under the infinite lattice constant due to lack of the interference effects the pulse will propagate with maximum velocity. Namely this fact was confirmed in consequence of the numerical analysis results. The pulse shape distortion should also be noted.

The following result deals with the dependence of both the shape and velocity of few-cycle pulses on modulation depth of nonlinear medium $\alpha$ and is shown in Fig. 4.

It should be noted that increase in modulation depth $\alpha$ leads not only to the pulse delay (for the reasons mentioned above) but to a change in its shape as well, due to the strong interference. The obtained results can also help to predict the value of pulse spreading when it slows down by means of nonlinear lattices in carbon nanotube media. 

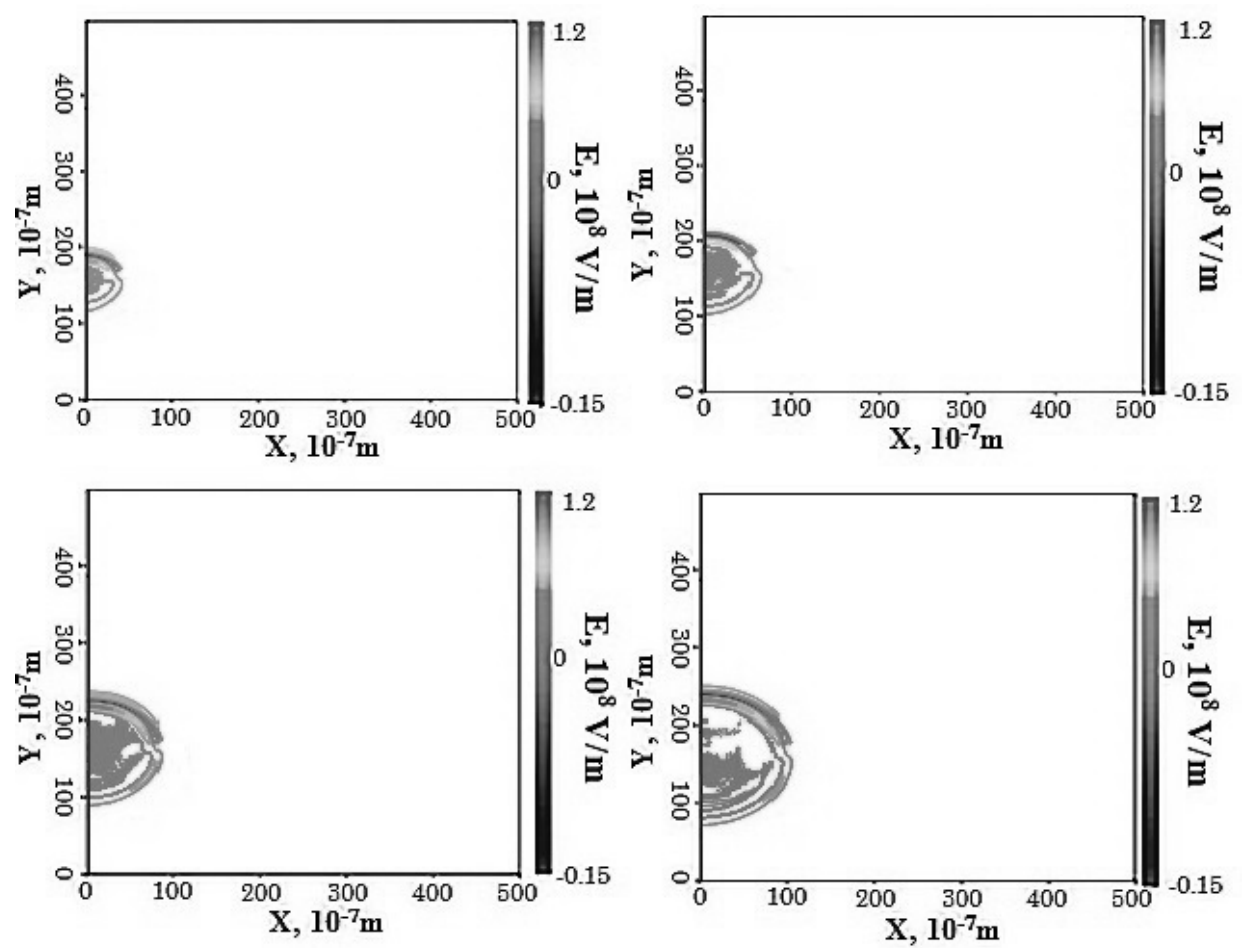

FIG. 2. Propagation of the light bullet in inhomogeneous medium of metallic CNTs $(\chi=$ $2.5 \mathrm{mcm}$ is the lattice constant, $\alpha=0.1$ is the modulation depth) at a fixed period of time T. A) $4 \times 10^{-12} \mathrm{~s}$; B) $6 \times 10^{-12} \mathrm{~s}$; C) $8 \times 10^{-12} \mathrm{~s}$; D) $10 \times 10^{-12} \mathrm{~s}$. The relative units of coordinates and electric field are along the axes
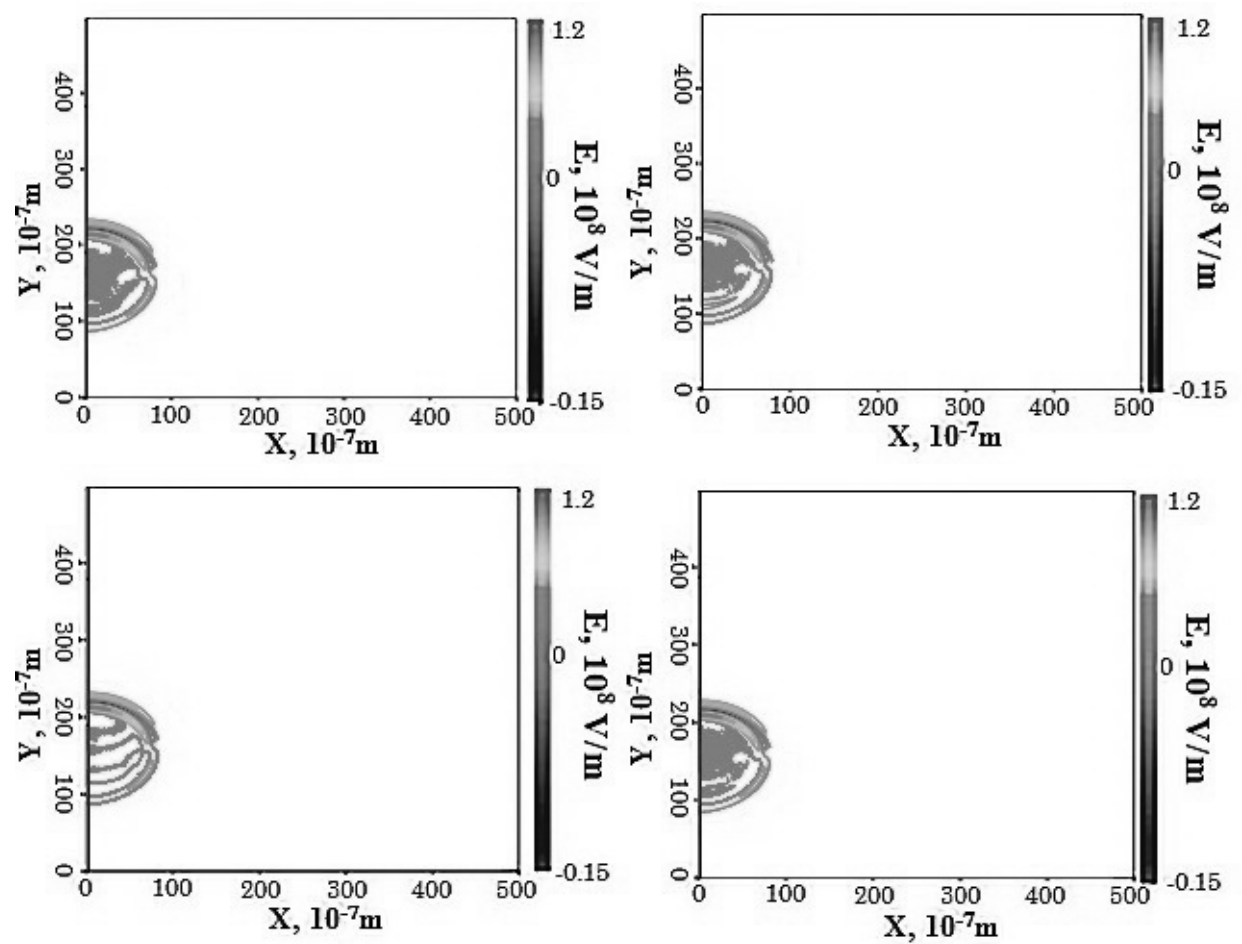

FIG. 3. Propagation of the light bullet in inhomogeneous medium of metallic CNTs at a fixed period of time $T=8 \times 10^{-12} \mathrm{~s}$ with different value of the lattice constant $\chi$ A) $0.125 \mathrm{mcm}$; B) $0.175 \mathrm{mcm}$; C) $0.225 \mathrm{mcm}$; D) $0.25 \mathrm{mcm}$. Relative units of coordinate and electric field are along the axes 

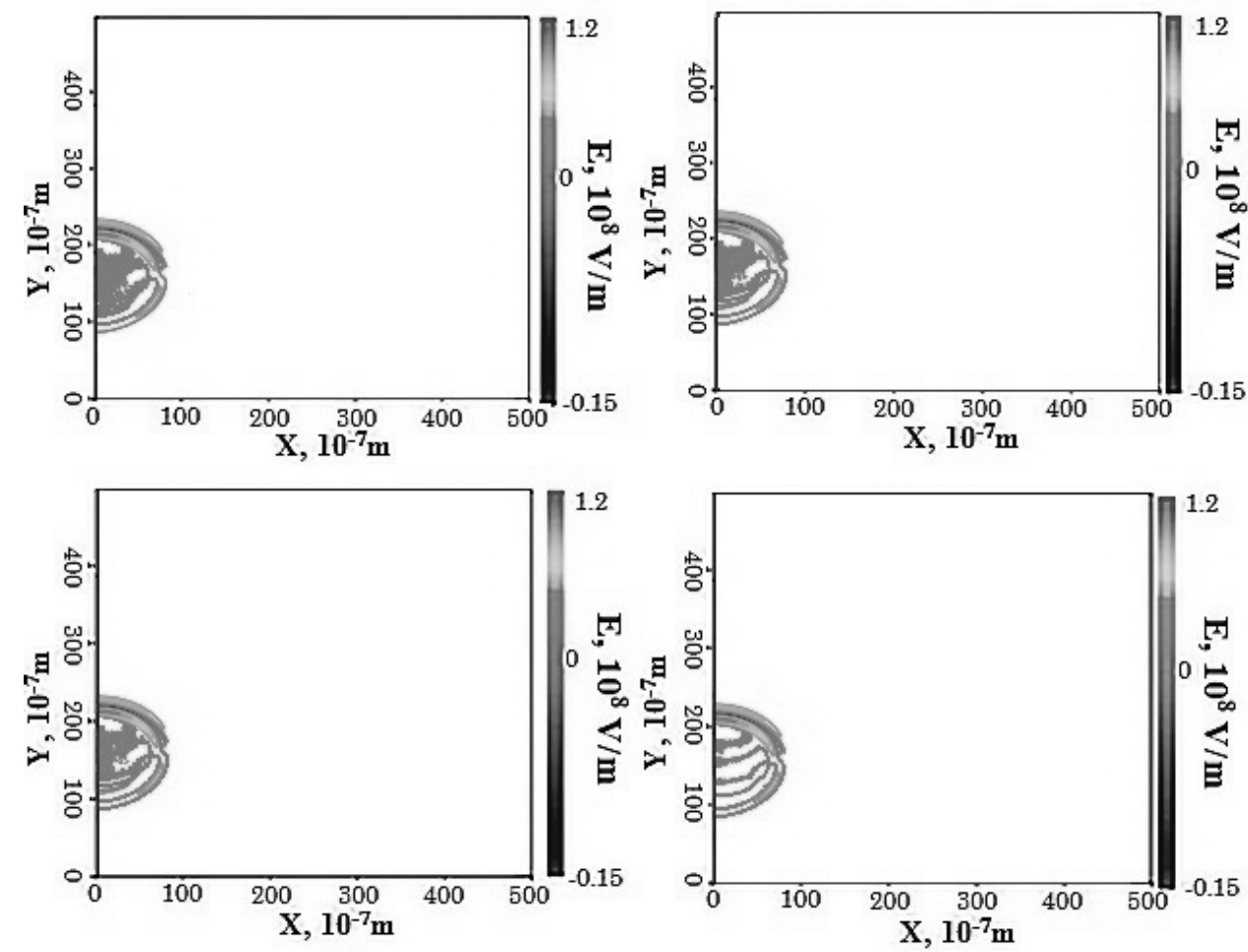

FIG. 4. Propagation of the light bullet in inhomogeneous medium of metallic CNTs $(\chi=5 \mathrm{mcm}$ is the lattice constant) at at fixed time with different modulation depth $\alpha$ A) $0.1 \alpha$; B) $0.3 \alpha$; C) $0.7 \alpha$; D) $0.9 \alpha$. The relative units of coordinates and electric field are along the axes

\section{Conclusion}

The performed investigation allows us to make the following conclusions:

1. The three-dimensional few-cycle optical pulse (light bullet) propagation in inhomogeneous medium with metallic carbon nanotubes is stable.

2. The conversion of CNT conductivity type from semiconductor to metallic one does not significantly affect the dynamics of three-dimensional few-cycle pulse propagation, which is of great importance for practical applications.

\section{References}

[1] Kaplan A.E., Shkolnikov P.L. Electromagnetic "bubbles" and shock waves: unipolar, nonoscillatig EM solitons. Physical Review Letters, 1995, 75(12), P. 2316-2319.

[2] Casperson L.W. Few-cycle pulses in two-level media. Phys. Rev. A., 1998, 57, P. 609.

[3] Brabec T., Krausz F. Intense few-cycle laser fields: Frontiers of nonlinear optics. Reviews of Modern Physics, 2000,72, P. 545-591.

[4] Schafer T. Wyane C.E. Propagation of ultra-short optical pulses in cubic-nonlinear media. Physica D., 2004, 196, P. 90-105.

[5] Kazantseva E.V., Maimistov A.I., Malomed B.A. Propagation and interaction of ultrashort electromagnetic pulses in nonlinear media with a quadratic-cubic nonlinearity. Optics communications, 2001, 188, P. 195-204.

[6] Kurizki G., Kozhekin A., Opatrny T., Malomed B. Optical solitons in periodic media with resonant and off-resonant nonlinearities. Progress in Optics E. Wolf, ed., (Elsevier, North-Holland), 2001, 42, P. 93-146.

[7] Maimistov A.I. Interaction of fast and slow varying electromagnetic waves propagating in paraelectric or ferroelectric material. Nanosystems: Physics, Chemistry, Mathematics, 2017, 8(3), P. 334-338.

[8] Saito R., Dresselhaus M.S., Dresselhaus G. Physical properties of carbon nanotubes. Imperial College Press, London, 1999,251 pp.

[9] Reich S., Thomsen C., Maultzsch J. Carbon nanotubes. Basic concepts and physical properties. Wiley-VCH Verlag, Berlin, 2003,218 pp.

[10] Harris P.J.F. Carbon nanotubes and related structures: New materials for the 21-st century. Cambridge University Press, Cambridge, 2009, $299 \mathrm{pp}$

[11] Belonenko M.B., Dvuzhilov I.S, Galkina E.N., Nevzorova Yu.V. Three dimensional few cycle optical pulses in nonlinear medium with carbon nanotubes. Modern Physics Letters B, 2016, 30(28), P. 1650345.

[12] Pavani S.R.P., Greengard A., Piestun R. Three-dimensional localization with nanometer accuracy using a detector-limited double-helix point spread function system. Appl. Phys. Lett., 2009, 95, P. 021103. 
[13] Spektor A.N.B., Shamir J. Singular beam microscopy. Appl. Opt., 2008, 47, P. A78-A87.

[14] Bozinovic N., Yue Ya., Ren Yo., Tur M., Kristensen P., Huang H., Willner A.E., Ramachandran S. Terabit-Scale Orbital Angular Momentum Mode Division Multiplexing in Fibers. Science, 2013, 340, P. 1545.

[15] Abramochkin E., Kotova S., Korobtsov A., Losevsky N., Mayorova A., Rakhmatulin M., Volostnikov V. Microobject manipulations using laser beams with nonzero orbital angular momentum. Laser Physics, 2006, 16, P. 842-848.

[16] Landau L.D., Lifshits E.M. Field theory (in Russian). Fizmatlit, Moscow, 1988, 536 pp.

[17] Bakhvalov N.S. Numerical Methods (Analysis, Algebra, Ordinary Differential Equations) (in Russian). Nauka, Moscow, 1975,632 pp. 